\title{
Carved Histories. Rotorua Ngati Tarawhai Woodcarving de Roger NEICH
}

\section{Gilles Bounoure}

\section{OpenEdition}

\section{Journals}

\section{Édition électronique}

URL : http://journals.openedition.org/jso/6747

DOI : 10.4000/jso.6747

ISSN : $1760-7256$

Éditeur

Société des océanistes

Édition imprimée

Date de publication : 31 décembre 2012

Pagination : 279-280

ISBN : 978-2-85430-033-8

ISSN : 0300-953x

Référence électronique

Gilles Bounoure, "Carved Histories. Rotorua Ngati Tarawhai Woodcarving de Roger NEICH », Journal de la Société des Océanistes [En ligne], 135 | 2012-2, mis en ligne le 26 janvier 2013, consulté le 24 septembre 2020. URL : http://journals.openedition.org/jso/6747 ; DOI : https://doi.org/10.4000/jso. 6747 
Miroux Daniel, 2011. Parlons iaai. Ouvéa, NouvelleCalédonie, Paris, L'Harmattan, 326 p., bibliogr., 2 cartes, quelques dessins noir et blanc.

Cette collection « Parlons...», qui compte plus de 170 ouvrages, a été conçue pour répondre aux besoins des voyageurs qui souhaitent acquérir les connaissances de base sur un peuple, sa culture et sa langue, en faisant de chaque volume une méthode d'autoapprentissage linguistique avec des éléments de conversation courante et deux lexiques. C'est donc ici un ouvrage grand public qui, de plus, n'est pas le fait d'un linguiste ou d'un anthropologue. En 326 pages, son auteur, économiste de formation mais connaisseur d'Ouvéa, propose ici une découverte d'Ouvéa. Dans l'introduction, une présentation générale de l'île, de son peuplement et de son histoire permet de situer la langue iaai par rapport à celle fagauvea introduite par les migrations wallisiennes anciennes (pp. 7-14). Puis, la première partie propose une description très simplifiée de la langue (pp. 15-34). La deuxième partie se veut comme un guide de conversation courante (pp. 35-46). Enfin, la troisième partie revient sur la culture (pp. 4752) et sur l'arrivée des Européens (pp. 53-72). Enfin, l'ouvrage se termine par les deux lexiques, françaisiaai (pp. 73-190) puis iaai-français (pp. 191-320). Une courte bibliographie termine l'ensemble (pp. 321-323).

L'auteur n'en est pas à son premier ouvrage sur la question. Il avait déjà publié à Nouméa à l'Alliance Champlain dont il est le président plusieurs ouvrages sur la langue iaai : un dictionnaire français-iaai Tusi hwen iaai ae gaan (en 2007) et un manuel de conversation en langue iaai Tusi hwen iaai (en 2003). Malheureusement, ces deux premiers volumes n'ont pas eu une grande diffusion en dehors de la NouvelleCalédonie et furent vite épuisés ; ce dernier ouvrage reprend donc une synthèse de ceux-ci dans une édition mieux diffusée en métropole. On ne peut donc qu'en saluer la parution. Les puristes de la langue et/ou de la culture pourront trouver l'ensemble trop vulgarisateur et plein d'imprécisions. Mais sans doute le but de cet ouvrage qui n'est pas scientifique est-il ailleurs et son utilité pour les néophytes sera importante. On le lit facilement et c'est une première approche de l'île et de la langue iaai. Ceux qui voudront en savoir plus pourront compléter leur lecture par celle des écrits de Françoise Ozanne-Rivierre qui a longuement travaillé en tant que linguiste sur cette langue et qui fut l'auteur notamment d'un dictionnaire (1984) et d'une description linguistique de la langue (1976).

\section{RÉFÉRENCES CITÉES}

OZANNE-RIVIERre Fançoise, 1976. Le iaai, langue mélanésienne d'Ouvéa (Nouvelle-Calédonie). Phonologie, morphologie, esquisse syntaxique, Paris, SELAF, $245 \mathrm{p}$.

—, 1984. Dictionnaire iaai-français (Ouvéa, NouvelleCalédonie), suivi d'un lexique français-iaai, Paris, SELAF, coll. Langues et cultures du Pacifique 6, 179 p.

Isabelle LEBLIC, LACITO CNRS, Villejuif
NeICH Roger, 2008. Carved Histories. Rotorua Ngati Tarawhai Woodcarving, Auckland, Auckland University Press, XVI-424 p., appendices, bibliogr., index, 19 pl. couleur, très nombreuses ill. noir et blanc dans le texte.

Dans cette nouvelle présentation comme dans sa première édition (2001) qui faisait suite à un précédent volume (Painted Histories, 1993) explorant à la fois les données livrées par les colons et celles issues des Māori amenés à se servir du médium de la peinture importé par les Européens, le présent ouvrage, considéré par de nombreux spécialistes comme le meilleur livre de Roger Neich (1944-2010), n'avait jamais été décrit dans ces colonnes. Il convient aussi de l'évoquer pour commencer à honorer le souvenir de ce savant infatigable et généreux, brutalement arraché aux travaux multiples qu'il continuait à mener sur les arts du Pacifique après sa retraite de professeur et de conservateur, et qui allaient souvent bien au-delà de ceux de la Nouvelle-Zélande dont il était devenu le principal expert, et le plus sollicité. Ces dernières années, n'avait-il pas enquêté avec succès sur les sculptures (conventionnellement dénommées tino) de Nukuoro, d'abord à partir d'une figuration masculine en mains privées dont il défendit publiquement l'authenticité sourdement contestée dans les milieux marchands (Neich, 2008 - véritable leçon d'expertise), puis sur les circonstances de collecte de la grande figuration féminine du musée d'Auckland, authentifiant au passage son identification à Kawe, « la principale déesse des insulaires de Nukuoro » (Neich and Park, 2009) ? Tel était son souci d'approfondissement et d'élargissement permanents des connaissances, qui le conduisait encore, sans la moindre " confusion des genres », à autoriser de grandes maisons de ventes à reproduire dans leurs catalogues les avis qu'il formulait sur des pièces offertes aux enchères, du fait de leur intérêt esthétique ou historique.

Les « Ngati Tarawhai, a small Rotorua tribe famed for their wood-carving prowess » comme les avait définis R. Neich antérieurement (1996: 76), ne figurent pas sur la plupart des « cartes simplifiées des tribus māori » offertes par les ouvrages spécialisés, leur région de la Bay of Plenty étant généralement assignée aux seuls Te Arawa, ses principaux premiers occupants. Elle compte dix-sept lacs (capables d'accueillir un championnat du monde de ski nautique en 2007) et R. Neich, enquêtant auprès des Tarawhai dès 1971, a reconstitué leur histoire ponctuée de migrations de l'un à l'autre de ces lacs et d'épidémies qui les décimèrent, avant que les guerres du XIX ${ }^{\mathrm{e}}$ siècle et leurs suites ne modifient largement leur style de vie (chapitres 2, 3, 4, pp. 4-41). À ces remarquables pages d'ethnohistoire succèdent trois chapitres consacrés aux sculpteurs de la tribu, à leur statut et à leurs contacts avec les sculpteurs Ngati Pikiao (terme désignant divers sousgroupes Te Arawa), avec lesquels ils collaboraient fréquemment (pp. 42-85).

Les chapitres 8, 9 et 10 (pp. 101-145) s'attachent à préciser ce que recouvrent aujourd'hui les termes d' " art » et de « style », par rapport aux contenus qui leur étaient attribués au XIX ${ }^{\mathrm{e}}$ siècle tant du côté māori 
que dans la conception que se faisaient les colons européens de l'art māori. Les deux suivants (pp. 146178) décrivent la sculpture māori sous l'angle de la culture matérielle, depuis ses outils et leur mise en Suvre jusqu'à ses réalisations les plus emblématiques, et du fait de leur portée générale et de leur profondeur, l'ensemble de ces pages mérite d'être connu de tous les spécialistes des arts du Pacifique. Leur succèdent trois chapitres $(13,14,15$, pp. 179-257) examinant et comparant, d'après ce qui subsiste des sculptures Tarawhai, ce qu'elles doivent à leurs commanditaires d'abord māori, puis européens, missionnaires ou fonctionnaires, et enfin touristes attirés un temps par les ressources thermales de Rotorua et de ses environs.

S'appuyant sur l'étude d'œuvres «individuelles » (connues comme telles ou attribuables au travail ou à la supervision d'un sculpteur Tarawhai unique, parfois anonyme), les quatre derniers chapitres (16-19, pp. 258-305) tentent de mesurer les changements survenus dans cet art d'abord du point de vue des motifs, des formes et des matériaux (un véritable changement " de langue », résume excellemment R. Neich), mais aussi dans les relations avec les commanditaires, et enfin dans les représentations mêmes que se sont successivement formées les sculpteurs Tarawahai de leur art et de leurs créations. Si la conclusion (pp. 306-308) appelant à de nouvelles recherches sur le sujet fait apprécier toute la modestie du savant, sa générosité à livrer les connaissances qu'il a recueillies, et son souci de les rendre facilement accessibles s'observent de nouveau avec ses neuf appendices (pp. 309-402), alternant précieux inventaires, développements historiques et thèmes de recherches impossibles à détailler ici, et avec les six index dont il a tenu à munir son livre (pp. 415-424).

Imprimé en petit corps, illustré de photographies que le lecteur renonce à compter tant elles se succèdent (parfois 6 par page) et sont inséparables des argumentations de R. Neich, cet épais volume est à ranger au nombre des classiques de l'histoire de l'art, non seulement pour l'abondance exceptionnelle de données et de réflexions qu'il offre au public en des termes la plupart du temps accessibles à tous, mais aussi pour sa méthode, qui a orienté et semble résumer le parcours de ce savant, réussissant à élargir sans cesse ses investigations et ses thèmes de recherche depuis ses premières enquêtes dans la région de Rotorua, et tenant en même temps à approfondir celles-ci jusqu'à ses dernières années. Mais est-ce là signaler cette publication d'un point de vue suffisamment « scientifique » et « critique » comme il est attendu dans ces colonnes ? Deux remarques, étayées sur des autorités dont $\mathrm{R}$. Neich fut le contemporain et devint rapidement l'égal, suffiront peut-être à dissiper ce genre de doute.

Terence Barrow (1923-2001), dans des publications qu'on ne saurait énumérer ici, a constamment signalé combien la dimension "érotique » des objets d'art polynésiens (et particulièrement māori dont il était grand spécialiste) s'était trouvée minorée ou occultée par les premiers Occidentaux qui mirent la main dessus pour les détruire ou les exporter (avec mutilation des sculptures), ou en firent exécuter des répliques plus conformes à la «pudeur». R. Neich aborde ce sujet (qui doit aussi être envisagé sous maints autres angles, familial, généalogique, politique...) avec une prudence et une rigueur qui appellent l'admiration, notamment à propos du motif des " couples enlacés 》 (embracing figures, pp. 280-282 pour le texte, pp. 280-285 pour les illustrations, et inventaire de ces figures dans l'appendice VIII pp. 396-398), dont il montre qu'il offrit surtout aux maîtres sculpteurs Tarawhai l'occasion de déployer leur virtuosité en expérimentant vues frontales ou de profil, membres ou parties du corps en commun, et figurations encore plus « ambiguës », amusantes ou déroutantes.

Un autre grand spécialiste des arts māori dont Carve d Histories conduit à évoquer les travaux, David R. Simmons, à qui R. Neich succéda comme conservateur des collections ethnologiques du musée d'Auckland, s'était attiré maintes critiques pour son empressement à assigner les objets à des styles régionaux précis en s'appuyant principalement sur leurs caractéristiques formelles, parfois arbitrairement ou confusément définies. Que cette tâche soit des plus difficiles, R. Neich le soulignait lui-même (1996, pp. 94-97) en faisant observer que dès la fin du XVIII ${ }^{\mathrm{e}}$ siècle apparaissent des objets où se mêlent plusieurs styles locaux, probablement du fait de l'intrusion des Occidentaux, surtout dans les régions côtières de l'île du Nord, celles des Arawa et des Tarawhai particulièrement. Le cheminement de ce volume suffit à l'indiquer, R. Neich n'a nullement rejeté l'ambition affichée par D. R. Simmons après d'autres (Best, Skinner, etc.), il lui a apporté la perspective et la rigueur historiques qui lui manquaient jusqu'alors. Sous cet aspect, qui fait pleinement comprendre le titre de Carved Histories, ce livre restera aussi comme une leçon de méthode exemplaire et comme un grand livre d'histoire.

\section{RÉFÉRENCES CITÉES}

NeICH Roger, 1996. Wood-carving, in D. C. Starzecka (ed.), Maori Art and Culture, London, British Museum Press, pp. 69-113.D

-, 2008. A recently revealed tino-aitu figure from Nukuoro Island, Caroline Islands, Micronesia, Journal of the Polynesian Society 117-4, pp. 327344.

NeICH Roger and Stuart PARK, 2009. A detailed provenance for Kawe, the Nukuoro figure carving in Auckland Museum, Journal of the Polynesian Society 118-4, pp. 369-375.

Gilles BOUNOURE

Starzecka Dorota C., Roger NeICH and Mick Pendergrast, 2010. Taonga Mãori in the British Museum, Wellington, Te Papa Press, viII-168 pages, appendices, bibliogr., index, 2 cartes, 24 planches couleur, 200 planches noir et blanc, autres ill. noir et blanc dans le texte.

Ni Roger Neich ni Mick Pendergrast, tous deux décédés en 2010, n'auront eu en mains ce livre, sorti 